\title{
Le BMS est et reste la revue de référence du corps médical
}

\section{Julia Rippstein}

Rédactrice print online

\begin{abstract}
Cet été, nous avons sondé l'avis de notre lectorat au sujet du Bulletin des médecins suisses (BMS). Les réponses qui nous ont été fournies nous encouragent à poursuivre notre travail et nous aident à continuer de proposer un contenu qui soit au plus proche des besoins des lectrices et lecteurs.
\end{abstract}

Le BMS est-il davantage lu en ligne ou sur papier? Quelle rubrique est la plus appréciée? Comment la qualité et la longueur des articles sont-elles jugées par le lectorat? Que pensent les internautes de notre site internet? Pour obtenir des réponses à ces questions et à d'autres, la rédaction du BMS a réalisé une enquête en ligne entre début juin et la mi-août 2020. Le questionnaire a été entièrement rempli par 542 personnes; 242 y ont répondu en partie, toutefois sans le terminer. Afin d'atteindre un maximum de personnes, nous proposions également la possibilité de remplir le questionnaire sur papier - ce support n'a toutefois pratiquement pas été sollicité. Si ce chiffre semble au premier abord modeste par rapport au tirage du journal (39000 exemplaires), les réponses fournies nous permettent de dégager des tendances qui seront utiles au développement du journal. Nous aimerions donc chaleureusement remercier toutes les participantes et tous les participants. Ils nous ont livré de précieuses informations.

\section{Numéro 1 en politique de santé}

Plus de 57\% des sondées ont répondu utiliser principalement le BMS pour s'informer sur le système de santé suisse. Pour près de $60 \%$, les articles du BMS aident à se forger une opinion sur les questions de politique de santé et de politique professionnelle. Le BMS est majoritairement lu sur papier: $51 \%$ préfèrent la version imprimée, tandis que $36 \%$ utilisent essentiellement la version en ligne. La version papier est d'ailleurs «agréable à lire» pour $63 \%$ des personnes sondées.

\section{Offre en ligne: potentiel d'amélioration}

Les résultats sont moins nets à propos du site internet (bullmed.ch). Les personnes se rendant sur le site consultent principalement «Numéro actuel» (62\%) ainsi que les archives (43\%). Près de la moitié des sondés affirme que celles-ci leur sont d'une grande aide. La

Pour $\mathbf{m}$ 'informer sur le système de santé suisse, j'utilise principalement...

Le Bulletin des médecins suisses (BMS)

Portails de certaines organisations (p. ex. OFSP, FMH, sociétés médicales,...)

Médias grand public (RTS, journaux quotidiens,....)

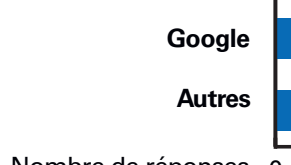

Nombre de réponses

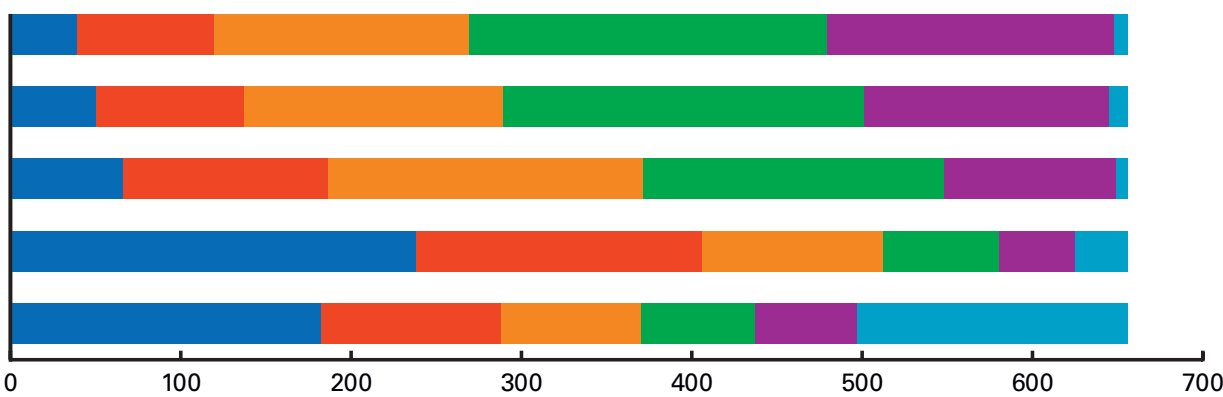

$\begin{array}{llllllll}1 & 2 & 3 & 4 & 5 & \text { Pas de réponse }\end{array}$

$1=$ ne correspond pas; $5=$ correspond tout à fait 


\section{Quel est votre intérêt pour les contenus suivants?}

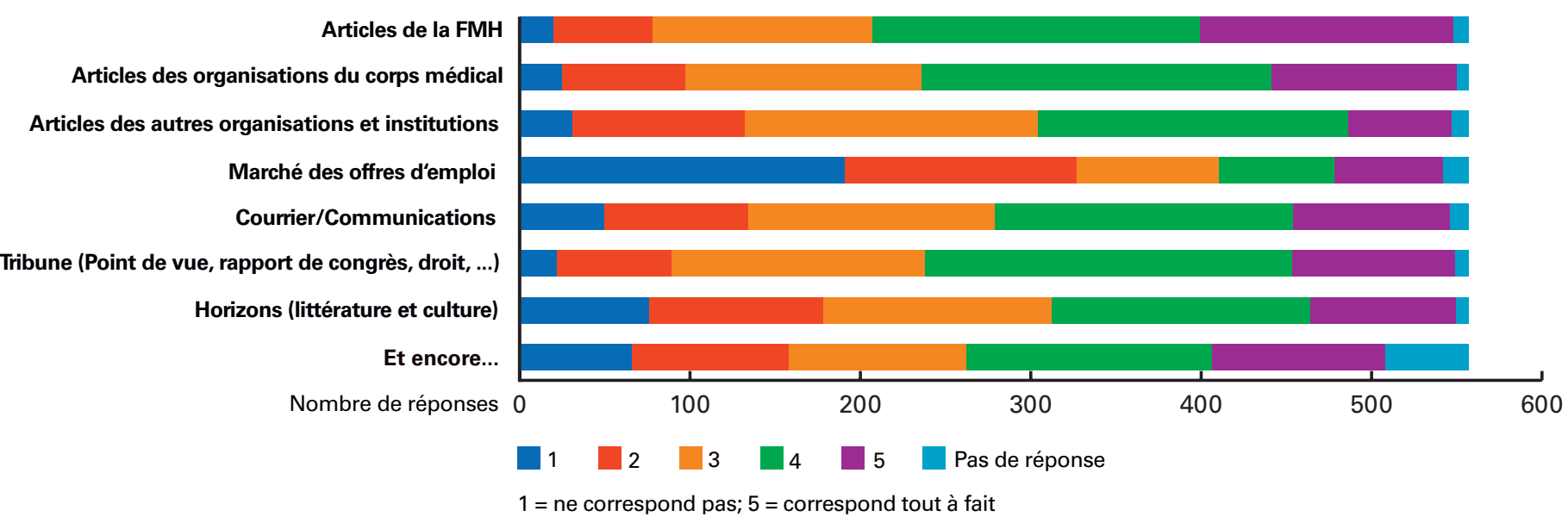

Figure 2: Mis à part les communications officielles des organisations, les textes d'opinion de la base et culturels sont aussi appréciés.

convivialité du site récolte un score moins bon: seuls $40 \%$ le trouvent agréable d'utilisation. Cet aspect fait l'objet de commentaires tels que «Die Seite erscheint veraltet» («Le site internet est désuet»), "Plutôt confus», «Könnte übersichtlicher gestaltet werden» («Pourrait être rendu plus clair»). Nous devons constater qu'il y a un important potentiel d'amélioration au niveau de notre offre en ligne.

\section{Goût pour la culture}

Quelles sont les rubriques du BMS les plus appréciées? Plus de $60 \%$ portent un grand intérêt aux textes de la FMH et les communications des sociétés médicales sont, elles aussi, beaucoup appréciées (56\%). Tout comme les communications officielles, les articles de la rubrique "Tribune» suscitent un fort intérêt chez près de $56 \%$ du lectorat. La culture n'est pas en reste: les textes publiés sous «Horizons» sont intéressants pour $42 \%$.

\section{Référence pour les médecins}

Qu'est-ce qui caractérise le BMS? Pour une majorité du lectorat, il s'agit de la revue de référence en matière d'informations officielles sur la politique professionnelle et la politique de santé. Quel est l'aspect le plus apprécié du BMS? En premier lieu, c'est la diversité des sujets, mais aussi le fait que le journal serve de plateforme de discussion et débat. La qualité, le sérieux et l'utilité des informations constituent aussi un point fort. Quant aux thèmes qui devraient être davantage abordés, les réponses sont très diverses: certains souhaiteraient encore plus de politique de santé, alors que d'autres aimeraient voir plus de textes scientifiques, juridiques voire philosophiques. Plusieurs ont émis le vœu d'entendre plus souvent la «base» et de lire des sujets plus actuels.

\section{«Continuez comme ça!», mais...}

La fin du questionnaire laissait carte blanche pour tout commentaire. La majorité des lectrices et lecteurs encourage notre rédaction à poursuivre son «bon travail» - ce qui nous a évidemment beaucoup réjoui. Il a toutefois été souligné à plusieurs reprises que les articles pourraient être qualitativement meilleurs et plus concis. Pour ce faire, nous allons devoir à l'avenir encore davantage réécrire les textes lors du processus d'édition. Un autre point à améliorer concerne la mise en page et l'aspect graphique du magazine. Parmi les Romands, plusieurs déplorent qu'il y ait trop d'articles en allemand dans la version francophone.

\section{Qui a répondu à l'enquête?}

Plus de deux tiers des personnes ayant répondu au sondage sont des hommes. L'âge est, lui, mieux réparti: on compte $30 \%$ de sondés de 36 à 54 ans, 31\% de 55 à 65 ans et $38 \%$ de plus de 65 ans. Malheureusement les plus jeunes (jusqu'à 35 ans) sont clairement sous-représentés (moins de 3\%).

Mis à part le corps médical, il est intéressant de constater que des représentantes et représentants d'autres domaines ont donné leur voix: droit, économie de la santé, conseil en communication dans le secteur médical, marketing, politique, recherche, direction d'en- 


\section{A quelle catégorie d'âge appartenez-vous?}

moins de 35 ans

$2.7 \%$

36-54 ans $27.8 \%$

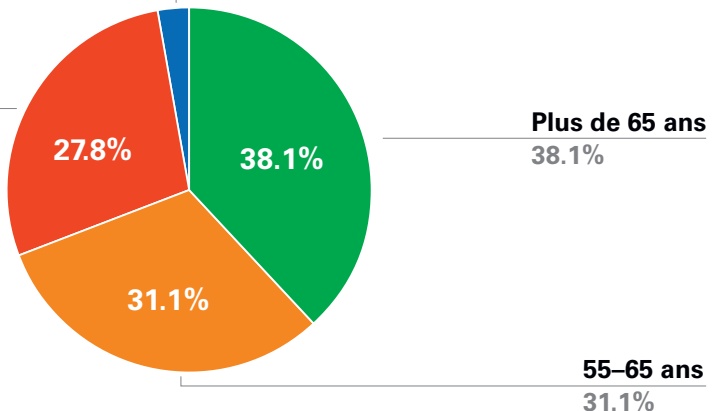

Figure 3: Seule une minorité de personnes de moins de 35 ans a participé au sondage.

treprise et d'hôpitaux, diététique, physiothérapie, personnel soignant. Une preuve que le BMS joue un rôle central pour les différents acteurs du système de santé.

\section{Conclusions}

D'un côté, les réponses obtenues confirment que le Bulletin des médecins suisses est considéré comme la «voix officielle» du corps médical et qu'il joue un rôle central en tant que plateforme de discussion de sujets de politique de santé. De l'autre, elles soulignent la nécessité d'adapter le contenu de la revue à l'évolution des besoins du lectorat: l'aspect graphique de la version papier et du site internet doit être remis au goût du jour, la prise en compte accrue lors du choix des sujets et des auteurs que les femmes constituent aujourd'hui la majorité du corps médical et, enfin, le contenu de journal doit aussi intéresser le public plus jeune. C'est désormais à nous, les Editions médicales suisses EMH, de mettre en œuvre ces changements. Où en sommesnous dans ce processus? La refonte du site est déjà en cours. Une adaptation de la mise en page de la version imprimée est également prévue. Quant à notre présence en Suisse romande, nous sommes très heureux d'accueillir au sein du comité de rédaction Anne-Françoise Allaz, non seulement une médecin très compétente, mais aussi une autre représentante de la partie francophone. Par ailleurs, nous nous efforçons depuis un an à proposer et à promouvoir des textes en français ainsi que de présenter des projets phares de Suisse romande.

Les jalons essentiels sont ainsi posés pour que le Bulletin des médecins suisses reste le fleuron de la politique professionnelle et de la santé.

Crédits graphiques

Celine Kohler EMH

\section{Connectez-vous} à myFMH
Changez votre adresse sous «Données personnelles»

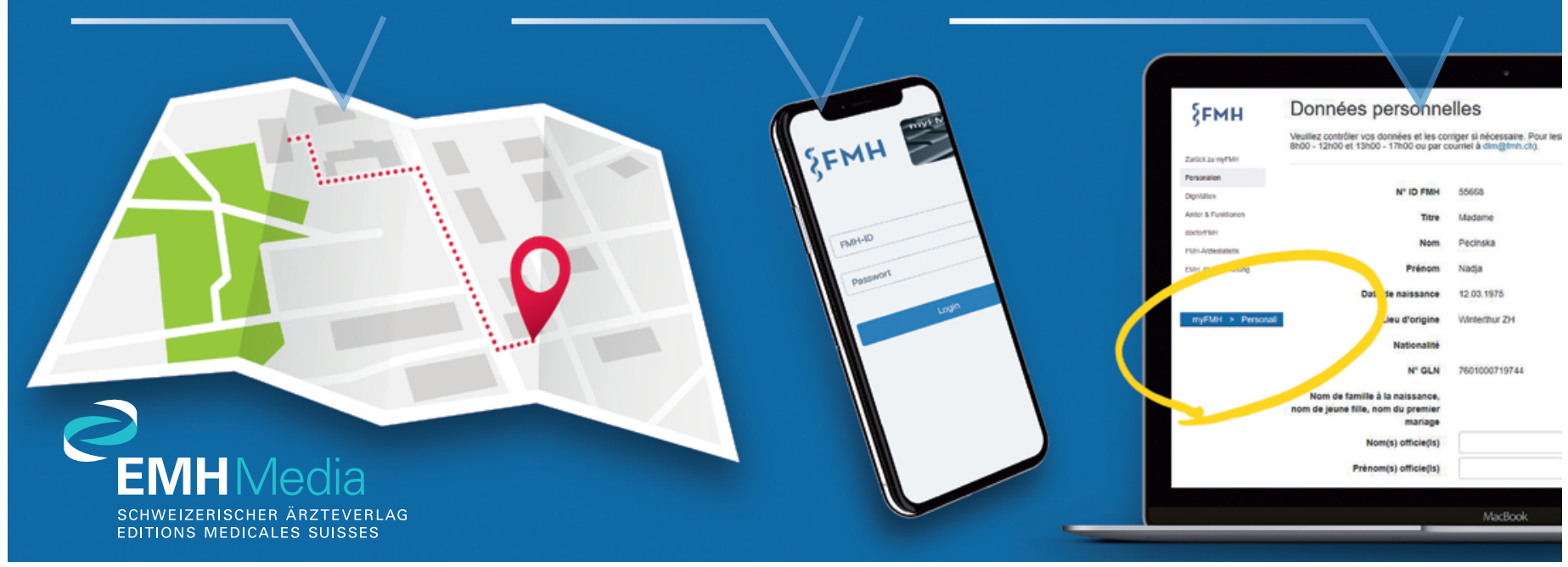

